\title{
Etude Ecologique Et Entomologique Des Culicides Larvaires Des Gites De La Province De Kenitra (Maroc)
}

\author{
Ikram Marc \\ Laboratoire de Biotechnologies, Environnement et Qualité. Faculté des \\ Sciences, Université Ibn Tofail. Kénitra, MAROC. \\ Laboratoire Provincial de Paludisme. \\ Délégation du Ministère de la Santé, Kénitra, MAROC \\ Abdelkader Chibani \\ Cellule Provinciale de Santé Environnement. \\ Délégation du Ministère de la Santé, Kénitra, MAROC \\ Ali Alemad \\ Arwa Alkhali \\ Asmaa Belala \\ Mohammed Hadji \\ Driss Belghyti \\ Khadija El kharrim
}

Laboratoire de Biotechnologies, Environnement et Qualité.

Faculté des Sciences, Université Ibn Tofail. Kénitra, MAROC

doi: 10.19044/esj.2016.v12n32p398 URL:http://dx.doi.org/10.19044/esj.2016.v12n32p398

\section{Abstract}

Mosquitoes are considered the source of nuisances and used as vectors of major pathogens for humans and animals. Despite the existence of many ways of control, supervising their populations remains a challenge forced by a delicate knowledge of their dynamics in space and time. Hence the need to conduct an entomological and ecological study of breeding sites in the province of Kenitra in order to acheive an inventory of culicidiennes species collected at eight stations of surface water with the measurement of physical-chemical parameters of these waters during several month from April to June 2012. The results revealed the presence of two species of mosquitoes, the Anopheles labranchiae and the Culex pipiens. The first type has a very low density at three stations, while the second one has a very high density in five stations. Furthermore, concentrations and measured values of the physico-chemical parameters allowed to highlight a correlation between the quality of waterin the stations and dynamics of mosquitoes. Thus, the 
Anopheles attend frequently the well-oxygenated and unpolluted stations $\left(\mathrm{O}_{2}\right.$ between 2,53 and 3,65 mg/l; BOD ${ }_{5}$ between 3 and $15 \mathrm{mg} / \mathrm{l}$ ) against the Culex (O2 between 3,85 and 8,12mg/l, $\mathrm{BOD}_{5}$ between 10 and $100 \mathrm{mg} / \mathrm{l}$ ).

Keywords: Entomology, Culicidae, Malaria, Kenitra, Morocco

\section{Résumé}

Les moustiques sont sources de nuisances et vecteurs d'agents pathogènes majeurs pour l'homme et les animaux. Malgré l'existence de moyens de lutte, la maîtrise de leurs populations reste un enjeu conditionné par une connaissance fine de leur dynamique dans l'espace et dans le temps. D'où l'intérêt de mener une étude entomologique et écologique des gîtes larvaires au niveau de la province de Kenitra. Cette étude comporte un inventaire des espèces culicidiennes récoltées au niveau de 8 stations d'eau de surface, avec la mesure des paramètres physico-chimique de ces eaux durant la période du mois d'avril au mois du juin 2012. Les résultats obtenus ont révélé la présence de deux espèces de moustiques: Anopheles labranchiae et Culex pipiens. La première espèce avec une très faible densité au niveau de trois stations, la deuxième espèce avec une très forte densité dans cinq stations. D'autre part, les concentrations et les valeurs des paramètres physico-chimiques mesurés ont permis de mettre en évidence une corrélation entre la qualité des eaux des stations et la dynamique des moustiques. Ainsi l'Anopheles fréquente des stations bien oxygénés et peu pollués $\left(\mathrm{O} 2\right.$ entre 2,53 et 3,65mg $/ / \mathrm{DBO}_{5}$ entre 3 et $\left.15 \mathrm{mg} / \mathrm{l}\right)$ par contre le Culex préfère des stations avec (O2 entre 3,85 et $8,12 \mathrm{mg} / \mathrm{l}$; DBO5 entre 10 et $100 \mathrm{mg} / \mathrm{l})$.

Mots clés. Entomologie, Culicidés, Malaria, Kénitra, Maroc

\section{Introduction}

Les maladies à transmission vectorielle figurent parmi les plus importantes (Becker \& al ., 2010) en santé humaine et animale (Paludisme, Dengue, Chikungunya, Fièvre du Nil, Fièvre jaune, Fièvre de la vallée du Rift, Encéphalites équines, Encéphalite japonaise, Encéphalite de Saint Louis). Elles sont transmises d'un individu infecté (un hôte vertébré : homme ou animal) à un autre par l'intermédiaire d'un arthropode hématophage (insecte ou acarien) (Rodhain \& Perez, 1985).

Leurs effets sont perceptibles non seulement sur la santé mais également sur le développement socio-économique des pays touchés, aussi bien par la morbidité que par la mortalité qu'elles entrainent chez l'Homme et les animaux. Les changements climatiques tels le réchauffement 
climatique, la modification des écosystèmes sont en faveur d'émergence ou réémergence des maladies à transmission vectorielle (Rodhain, 2000).

Afin d'améliorer la compréhension et le contrôle de ces maladies vectorielles, différents modèles peuvent être développés dont deux principaux: les modèles géographiques, pouvant être utilisés pour décrire la distribution des vecteurs en fonction de conditions environnementales (Clements, 1999; Clements, 2000; Becker et al., 2010) et les modèles épidémiologiques liés au calcul de la capacité vectorielle et aux taux de reproduction de base de la maladie (Tran et al., 2005).

Dans le présent travail on se propose de réaliser une étude à double objectif, entomologique et écologique sur les populations culicidiennes du gharb.

\section{Milieu d'étude}

La province de Kenitra fait partie de la région du Gharb-ChrardaBeni Hssen et se situe au Nord-Ouest du pays. Sa superficie est de $4745 \mathrm{~km}^{2}$ avec une côte de $140 \mathrm{~km}$ sur l'atlantique. Sa population est estimée à 1167301 habitants répartie avec des taux de 49,06 \% en milieu urbain et $50,09 \%$ en milieu rural (RGPH, 2004). Le climat de la région est de type méditerranéen, caractérisé par l'alternance d'une saison humide d'Octobre à Avril et une saison sèche et chaude de Mai à Septembre, avec une moyenne des températures de $24^{\circ} \mathrm{C}$. Les précipitations se situent autour d'une moyenne de $450 \mathrm{~mm}$ par an (HCP, 2008).

La province de Kenitra se caractérise par la présence d'un potentiel hydrique superficiel très important auquel s'ajoute une diversité de la végétation aquatique (HCP, 2008). L'ensemble de ces facteurs favorise la multiplication des gîtes larvaires de plusieurs moustiques vecteurs d'un grand nombre de maladies humaines, animales et source de nuisance (Brahmi \& al., 2007).

Les stations (S1,S2,S3,S4,S5,S6,S7 et S8) qui ont fait l'objet de l'étude ont été choisies de façon à avoir une représentativité, en tenant compte de leurs caractères écologiques à savoir milieu urbain, périurbain, rural, présence de l'eau permanente ou saisonnière, eau courante ou stagnante (Figure1) . 


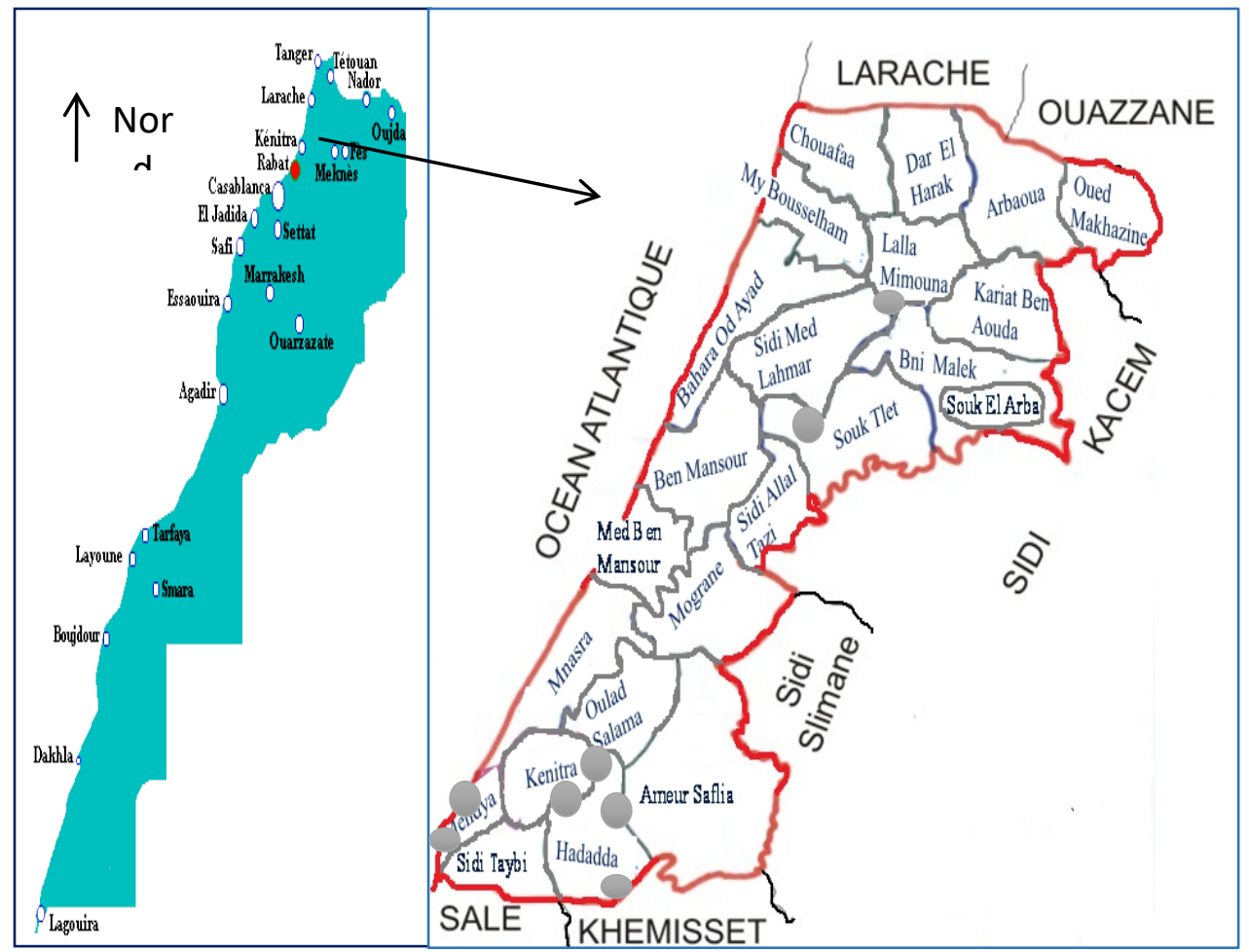

Figure 1: Localisations géographiques des huit sites d'étude.

\section{Méthodes d'étude}

Les larves de moustiques sont récoltées au niveau des grandes collections d'eau à l'aide d'un filet troubleau qui est constitué d'un cercle de fer de $30 \mathrm{~cm}$ de diamètre sur lequel est montée une poche en toile de tulle permettant l'écoulement de l'eau tout en retenant les larves. Un manche de 2 $\mathrm{m}$ en bois ou en canne de fer s'adapte au cercle par une douille (Bameul, 1990). Pour les petites collections d'eau, la collecte des larves est réalisée à l'aide de louches d'une contenance de $500 \mathrm{ml}$ (Bendali \& al., 2001).

La détermination des espèces culicidiennes a été faite au Laboratoire de Biotechnologie, Environnement et Qualité. On a utilisé une loupe binoculaire, un microscope optique et le logiciel d'identification des moustiques de l'Afrique méditerranéenne (Brhunes et al., 1999) qui permet l'identification en se basant sur un ensemble de critères et de descripteurs microscopiques très précis.

Les paramètres physico-chimiques des eaux des stations à savoir: la température, le $\mathrm{pH}$, le potentiel d'oxydo-reduction, la conductivité électrique, la résistivité, TDS, la salinité, turbidité et oxygène dissous ont été mesuré à l'aide d'un analyseur multi-paramètre. $\mathrm{La}^{\mathrm{DBO}} 5$ a été mesurée à l'aide d'un DBO mètre (Rodier, 1984,1996). 


\section{Résultats et discussion}

L'identification morphologique des larves récoltées au niveau des différentes stations a révélé l'existence de deux espèces culicidiennes réparties en deux genres : Anopheles et Culex (Tableau1) :

- Anopheles labranchiae au niveau des stations S4, S5 et S6 pendant le mois de mai avec une faible densité en avril;

- Culex pipiens au niveau des stations S1, S2, S3, S4 et S5 avec une forte densité pendant le mois de juin;

- Absence de larves de moustiques au niveau de deux stations S7 et S8 durant toute la période d'étude.

Tableau 1: Distribution des espèces larvaires et caractéristiques écologiques des gîtes.

\begin{tabular}{|c|c|c|c|c|c|c|c|c|}
\hline & S1 & S2 & S3 & S4 & S5 & S6 & S7 & S8 \\
\hline Culex & + & + & + & + & + & 0 & 0 & 0 \\
\hline Anopheles & 0 & 0 & 0 & + & + & + & 0 & 0 \\
\hline Végétation & Typha & Typha & Roseau & $\begin{array}{c}* \text { Hydro- } \\
\text { cotile } \\
* \text { Ranun- } \\
\text { culus }\end{array}$ & $\begin{array}{c}* \text { Ranun- } \\
\text { culus } \\
* \text { Stenotap- } \\
\text { hrum }\end{array}$ & $\begin{array}{c}* \text { Ranun- } \\
\text { Culus } \\
* \text { Stenota- } \\
\text { Phrum }\end{array}$ & Typha & Typha \\
\hline $\begin{array}{c}\text { Faune } \\
\text { Vertébrés }\end{array}$ & Orgileux & Argileux & Tirs & $\begin{array}{c}\text { Argileux } \\
\text { sablleux }\end{array}$ & Tirs & Tirs & Sableux & Sableux \\
\hline
\end{tabular}

Tableau 2 : Valeurs moyennes des mesures physicochimiques relevées dans les stations d'étude.

\begin{tabular}{|c|c|c|c|c|c|c|c|c|}
\cline { 2 - 9 } \multicolumn{1}{c|}{} & \multicolumn{9}{c|}{ Stations } \\
\hline $\mathrm{T}^{\circ} \mathrm{C}$ & $\mathrm{S} 1$ & $\mathrm{~S} 2$ & $\mathrm{~S} 3$ & $\mathrm{~S} 4$ & $\mathrm{~S} 5$ & $\mathrm{~S} 6$ & $\mathrm{~S} 7$ & $\mathrm{~S} 8$ \\
\hline Redox mv & $-22,6$ & 23,7 & 23,4 & 24,9 & 23 & 22,9 & 22,2 & 22,9 \\
\hline $\mathrm{Ph}$ & 7,4 & 7,4 & 8,14 & 6,89 & 6,7 & 6,48 & 8,2 & 8,23 \\
\hline $\mathrm{O} 2 \mathrm{mg} / \mathrm{l}$ & 3,85 & 6,7 & 8,12 & 2,53 & 3,65 & 3,65 & 0,48 & 0,54 \\
\hline Résistivité mV & 1,05 & 0,77 & 1,55 & 2,4 & 11 & 5,6 & 0,1 & 0,1 \\
\hline $\mathrm{CE} \mu \mathrm{s} / \mathrm{cm}$ & 934 & 1263 & 628 & 435 & 100 & 174 & 9400 & 7440 \\
\hline TDS mg/l & 478 & 646 & 323 & 203 & 54 & 92 & 4166 & 3473 \\
\hline Sal g/l & 0,47 & 0,64 & 0,31 & 0,19 & 0,09 & 0,09 & 4,84 & 3,8 \\
\hline Turbidité NTU & 46,5 & 41,2 & 26 & 21,7 & 6,5 & 7,8 & 51,9 & 94,5 \\
\hline DBO ${ }_{5} \mathrm{mg} / \mathrm{l}$ & 48 & 100 & 10 & 4 & 3 & 15 & 28 & 35 \\
\hline Culex & 34 & 42 & 17 & 5 & 2 & 0 & 0 & 0 \\
\hline Anophèle & 0 & 0 & 0 & 7 & 19 & 30 & 0 & 0 \\
\hline
\end{tabular}


Tableau 3: Mesures physicochimiques relevées dans les gîtes suivies.

\begin{tabular}{|c|c|c|c|c|}
\hline Variable & Minimum & Maximum & Moyenne & Ecart-type \\
\hline $\mathrm{T}^{\circ} \mathrm{C}$ & 22,20 & 24,90 & 23,31 & 0,86 \\
\hline Redox mV & $-68,40$ & 29,90 & $-18,90$ & 38,90 \\
\hline $\mathrm{pH}$ & 6,48 & 8,23 & 7,33 & 0,69 \\
\hline $\mathrm{O}_{2} \mathrm{mg} / \mathrm{l}$ & 0,48 & 6,70 & 3,06 & 2,15 \\
\hline Résistivité mV & 0,10 & 11,00 & 3,00 & 4,01 \\
\hline $\mathrm{CE} \mu \mathrm{S} / \mathrm{cm}$ & 100,00 & 9400,00 & 2820,86 & 3888,23 \\
\hline $\mathrm{TDS} \mathrm{mg} / \mathrm{l}$ & 54,00 & 4166,00 & 1301,71 & 1744,25 \\
\hline Sal g/l & 0,09 & 4,84 & 1,45 & 2,00 \\
\hline Turbidité NTU & 6,50 & 94,50 & 38,59 & 30,65 \\
\hline $\mathrm{DBO}_{5} \mathrm{mg} / \mathrm{l}$ & 3,00 & 100,00 & 33,29 & 33,68 \\
\hline
\end{tabular}

La température de l'eau des gîtes étudiés est comprise entre 22,2 et $24,9^{\circ} \mathrm{C}$ favorable au développement de plusieurs espèces de moustiques. Pour une température maintenue entre 23 et $26^{\circ} \mathrm{C}$, la durée du cycle de développement pré-imaginal d'Anopheles labranchiae varie entre 10 et 13 jours (Laboudi \& al., 2014), et de 10 à 14 jours pour Anopheles arabiensis. Tandis que chez Anopheles gambiae, la durée du cycle de développement est de 15,8 jours en moyenne (Diop \& al., 1998).

Le $\mathrm{pH}$ de l'eau des stations 4, 5 et 6 est légèrement acide, mais pour l'ensemble des stations les valeurs de ce paramètre sont comprises entre un minimum 6,48 et un maximum 8,23 qui sont incluses dans la fourchette 6,5 et 8,5 de la classe excellente des eaux de surfaces selon la grille de qualité des eaux de surface (MATUHE, 2002). Les stations S7 et S8 sont situées au lac Sidi Boughaba, leurs $\mathrm{pH}$ est alcalin de valeur respectivement 8,2 et 8,23. ces valeurs sont similaires à celles trouvés par (Lachhab \& al., 2013) entre juillet 2009 et mai 2010 au niveau de ce lac.

Le $\mathrm{pH}$ de l'eau reflète également l'activité biologique du milieu. Une alcalinisation du milieu peut être causée par une hausse de l'activité photosynthétique dans le lac (Serghini et al., 2010).

On note une certaine corrélation entre le $\mathrm{pH}$ des eaux et le genre de moustique identifié à savoir que l'Anopheles préfère le $\mathrm{pH}$ acide et le Culex préfère les $\mathrm{pH}$ neutres et basiques. Il semble que le $\mathrm{pH}$ d'environ 6,4 soit limitant et empêche l'installation des larves de Culex d'après nos résultats trouvés.

Le potentiel d'oxydo-reduction représente la capacité d'oxydation ou de réduction d'une solution ; lorsqu'il est faible ou négatif, il entend une capacité importante qui est synonyme d'une intense eutrophisation du lac (Golterman, 1975). Dans notre étude, les larves de moustiques récoltées des gîtes qui ont un potentiel redox négatif sont des larves de Culex, Par contre 
les larves d’Anophèles identifiées sont récoltées des gîtes qui ont un potentiel redox positif.

La conductivité des eaux des gîtes étudiés est très élevée pour S7 et S8; elle dépasse $7400 \mu \mathrm{S} / \mathrm{cm}$ avec absence totale des larves de moustiques. Au niveau des stations S1, S2 et S3 la conductivité des eaux mesurées est comprise entre 600 et $1300 \mu \mathrm{S} / \mathrm{cm}$, on note la présence des larves de Culex. Par contre, les trois stations restantes S4, S5 et S6 ont une conductivité inférieure à $440 \mu \mathrm{S} / \mathrm{cm}$ et les larves identifiées sont celles d'Anopheles.

Les teneurs de la $\mathrm{DBO}_{5}$ sont respectivement de $4 \mathrm{mg} / \mathrm{l}$ et $3 \mathrm{mg} / \mathrm{l}$ au niveau de S4 et S5. Pour les stations S3 et S6 sont situées entre 10 et 15 $\mathrm{mg} / \mathrm{l}$, Par contre S1, S2, S7 et S8 ont des valeurs comprises entre $28 \mathrm{mg} / \mathrm{l}$ et $100 \mathrm{mg} / \mathrm{l}$. Selon Martin (1979), une $\mathrm{DBO}_{5}$ qui dépasse la valeur $10 \mathrm{mg} / \mathrm{l}$ représente une situation anormale d'où l'importance des nuisances causées par les matières organiques.

Les valeurs de la salinité sont inferieures à $0,1 \mathrm{~g} / \mathrm{l}$ au niveau de S5 et S6, pour S4 elle est de 0,19 g/l. On a observé une légère augmentation au niveau de S1, S2 et S3 avec des concentrations comprises entre 0,3 et 0,5 g/l. Une forte concentration est détectée au niveau de S7 et S8 avec des valeurs respectivement de 3,8 et 4,9 g/l. On note une variation considérable d'un lieu à un autre et seuls les eaux de S7 et S8 sont classées comme eaux salines vue leurs concentrations qui sont comprises entre $1 \mathrm{~g} / \mathrm{l}$ et $35 \mathrm{~g} / \mathrm{l}$ (Kharaka et Hanor, 2005).

D’après les résultats (Tableau 2), on conclut que la salinité, la conductivité et les TDS sont corrélées et influent sur l'espèce de moustique. Nous insistons sur le fait que dans les stations S7 et S8 les fortes teneures de ces 3 paramètres par rapport aux autres stations éliminent aussi bien les Culex que les Anopheles. La baisse de la salinité favorise la prolifération des espèces aquatiques (Laleye, 2010).

L’analyse en composantes principales (ACP) des données (Tableau 4 et 5, Figure 2 et 3) montre que les pourcentages d'inertie des deux premiers axes principaux totalisent $87,3 \%$ d'information sur la distribution des variables physico-chimiques des stations étudiées.

Tableau 4: Pourcentages d'inertie des deux premiers axes principaux.

\begin{tabular}{|c|c|c|c|c|c|c|}
\hline & F1 & F2 & F3 & F4 & F5 & F6 \\
\hline Valeur propre & 6,73 & 2,00 & 0,90 & 0,22 & 0,12 & 0,03 \\
\hline Variabilité (\%) & 67,27 & 20,03 & 8,98 & 2,20 & 1,23 & 0,29 \\
\hline \% cumulé & 67,27 & 87,30 & 96,28 & 98,48 & 99,71 & 100,00 \\
\hline
\end{tabular}


Tableau 5: Corrélations entre les variables et les facteurs.

\begin{tabular}{|c|c|c|c|c|c|c|c|}
\hline & & F1 & $\mathrm{F} 2$ & F3 & $\mathrm{F} 4$ & F5 & F6 \\
\hline \multicolumn{2}{|c|}{$\mathrm{T}^{\circ} \mathrm{C}$} & $-0,52$ & 0,44 & 0,71 & 0,01 & 0,17 & $-0,04$ \\
\hline Redox & $\mathrm{Mv}$ & $-0,97$ & $-0,21$ & $-0,01$ & $-0,07$ & $-0,11$ & $-0,08$ \\
\hline \multicolumn{2}{|c|}{$\mathrm{Ph}$} & 0,97 & 0,18 & 0,01 & 0,08 & 0,09 & 0,09 \\
\hline $\mathrm{O} 2$ & $\mathrm{mg} / \mathrm{l}$ & $-0,67$ & 0,64 & $-0,36$ & $-0,02$ & 0,08 & $-0,01$ \\
\hline Résistivité & $\mathrm{mV}$ & $-0,69$ & $-0,56$ & $-0,31$ & 0,27 & 0,19 & $-0,03$ \\
\hline $\mathrm{CE}$ & $\mu \mathrm{S} / \mathrm{cm}$ & 0,97 & $-0,19$ & $-0,05$ & $-0,10$ & 0,07 & $-0,05$ \\
\hline TDS & $\mathrm{mg} / \mathrm{l}$ & 0,98 & $-0,17$ & $-0,05$ & $-0,07$ & 0,06 & $-0,06$ \\
\hline Sal & $\mathrm{g} / \mathrm{l}$ & $\mathbf{0 , 9 7}$ & $-0,20$ & $-0,06$ & $-0,10$ & 0,07 & $-0,05$ \\
\hline Turbidité & NTU & $\mathbf{0 , 8 7}$ & 0,28 & 0,14 & 0,33 & $-0,14$ & $-0,06$ \\
\hline DBO5 & $\mathrm{mg} / \mathrm{l}$ & 0,21 & 0,91 & $-0,36$ & $-0,02$ & 0,02 & $-0,04$ \\
\hline
\end{tabular}

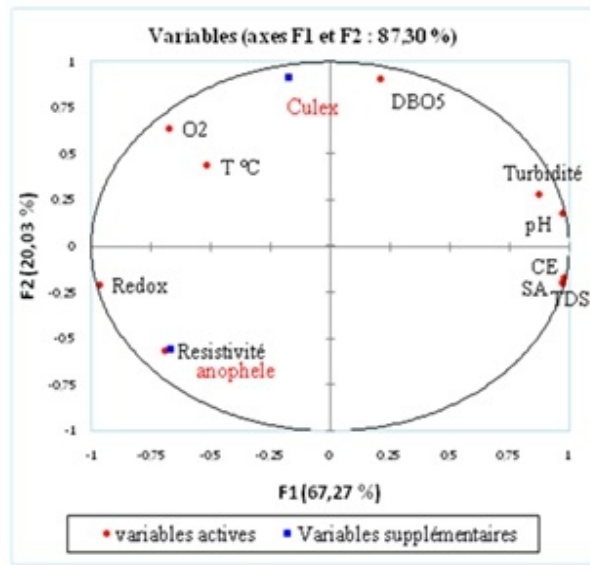

Figure 2: Projection du cercle de corrélation des variables sur le plan F1 x F2.

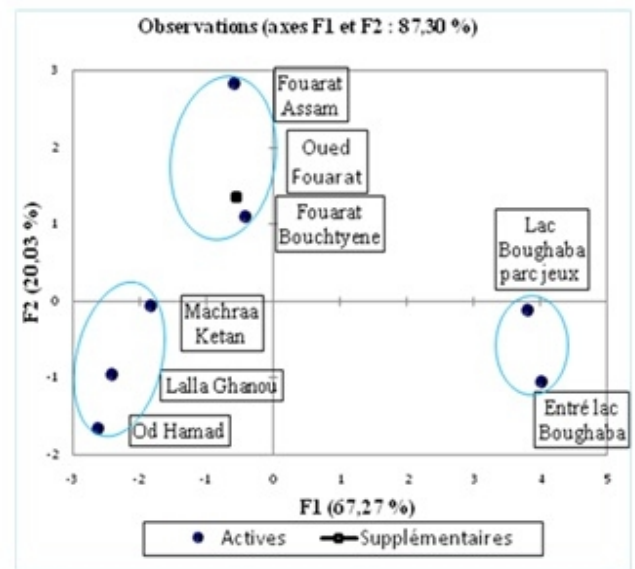

Figure 3: Dstribution des stations sur le plan factoriel F1 x F2.

La projection des paramètres physico-chimiques (Tableau 5) sur le plan factoriel F1×F2 montre les résultats ci-dessous :

- $\quad$ L’axe F1 est corrélé essentiellement avec le pH, la conductivité, la salinité et la turbidité;

- $\quad$ L'axe F2 est corrélé avec le $\mathrm{DBO}_{5}$ et l' $\mathrm{O}_{2}$.

La projection des variables sur le cercle de corrélation montre l'individualisation de 3 groupes $\mathrm{pH}$, turbidité, $\mathrm{CE}$, Sal, TDS; le groupe $\mathrm{DBO}_{5}, \mathrm{~T}^{\circ} \mathrm{C}$ et oxygène dissout et le groupe Redox, Résistivité.

La projection des relevés sur le plan F1 x F2 permet de distinguer aussi 3 groupes :

- $\quad$ Un $1^{\text {er }}$ groupe composé par S7 et S8 caractérisé par des fortes teneurs en conductivités, salinité, turbidité et DBO5; 
- $\quad$ Un $2^{\text {ème }}$ groupe constitué par S1, S2 et S3 caractérisé par des teneurs élevées en oxygène, et $\mathrm{pH}$ légèrement basique;

- $\quad$ Un $3^{\text {ème }}$ groupe formé par le gîte S4, S5 et S6 caractérisé par une faible teneur en conductivité, DBO5, salinité et pH légèrement acide.

\section{Conclusion}

Les espèces identifiées morphologiquement durant cette période d'avril à juin 2012 au niveau des 8 stations surveillées sont Anopheles labranchiae et Culex pipiens. Les résultats obtenus révèlent l'impact des paramètres physico-chimiques et les facteurs environnementaux sur la répartition et l'existence des populations larvaires de moustiques.

Nos résultats concordent avec ceux de (Kbibch \& al., 2012), (Messai \& al., 2011) et (Brahmi \& al., 2007) qui ont jugé que le biotope primordial pour la prolifération de moustiques résulte de l'interaction entre plusieurs composantes environnementales comme l'eau, les sols hydro morphes et salés et la végétation qui leur est associée.

Pour la capacité d'adaptation de l'espèce avec les conditions du milieu nos résultats sont en accord avec ceux exprimés par (Savage \& Miller, 1995) qui ont prouvé que les larves de Culex pipiens se retrouvent dans les divers gîtes des milieux urbains et périurbains, plus particulièrement ceux riches en matières organiques.

Par contre, les Anopheles, d’après nos résultats, préfèrent des milieux qui ont une faible salinité et DBO5 avec un pH légèrement acide. Donc on déduit que la pullulation des espèces de moustiques est conditionnée par la nature du gîte, ainsi (Alami \& al., 2010) ont indiqué que la nature du gîte larvaire favorise l'une ou l'autre espèce selon les caractéristiques du gîte : stagnant ou courant, pollué ou non, dépourvu ou riche en végétation.

\section{References:}

1. Kbibch, A., Elkharim, K., Elkhokh, K., Chentoufi, M., et Belghyti, D. (2012). Etude de l'impact des eaux usées de la ville de Souk el Arbaa sur la prolifération des moustiques. (Culex pipiens).

2. Bailly, H., et Choumara, A. (1968). Contribution à l'étude des moustiques du Maroc. Cah. ORSTOM, see. Ent. med., vol. VI, n 2.

3. Bameul, F. (1990). Le filet troubleau. In ancêtre d'insecte, 7p.

4. Becker, N., Petric, D., Zgomba, M., Boase, C., Madon, M., Dahl, C., et Kaiser, A. (2010). Mosquitoes and Their Control, Second edition Springer, edition 2010.

5. Bendali, F., Djebbar, F., et Soltani, N. (2001). Efficacité comparé de quelques espèces de poisson à l'égard de divers stades de Culx pipiens Larvaire dans des conditions de laboratoire. Parasitica. 57 (4) 255-265. 
6. Brahmi, N., Hatira, A., Rabia, M. C., Tounsi, I., Bouhafa, T., et baccar, L. (2007). Cartographie des zones à risque de prolifération des Aedes : application de l'approche géomatique dans la région de Bizerte (Tunisie). Revue Méditerranéenne de l’Environnement (2) pp 397-413.

7. Clements, A. N. (1999). The Biology of Mosquitoes: Sensory, Reception, and Behaviour.

8. CABI Publishing, Eastbourne.

9. Diop, A., Faye, O., Molez, J.F. (1998). Mise en place d'un insectarium d'une souche d'Anophèles arabiensis(Diptera : Culicidae),manuscrite $n^{\circ} 191$ Entomologie médicale

10. El oualilalami, A., El hilali , O., Benlamlih, M., Merzouki, M., Raiss, N., Ibensouda-kouraichi, S., \& Himmi, O. (2010). Etude entomologique, physico-chimique et bactériologique des gîtes larvaires de localités à risque potentiel pour le paludisme dans la ville de Fès. Bulletin de l'Institut Scientifique, Rabat, 32 (2): 119 127

11. Golterman, H.L. (1975). Développement in water science, 2.Physicalogical limnology.

12. Royaume du Maroc Haut Commissariat au Plan Monographie régionale 2008

13. IRD. (1999). Clé de détermination des Culicidae d'Afrique méditerranéenne : Institut de Développement et de Recherche. Support informatique Cd Rome.

14. Kharaka, Y. K., Hanor, J. S. ( 2005). Deep fluids in the continents: I sedimentary basins. Treatise on Geochemistry; : 499-540

15. Lachhab, M., El kharrim, K. , El Abidi, A. , Ben Akkame, R., Belghyti , D. (2013) . Etude physico-chimique des eaux du lac Sidi Boughaba - Site Ramsar - Kénitra Maroc

16. Laleye, P.( 2010). Biodiversité et exploitation des ressources vivantes aquatiques du Bénin : état des lieux. Revue Scientifique du CBRS.

17. Louah, M.A.(1995). Ecologie des Culicidae (Diptères) et état du paludisme dans la péninsule de Tanger, Thèse d'état Es-sciences Fac. Sc. Tétouan.

18. Laboudi. Majda., Sadak . Abderrahim, ,Faraj, Chafika . (2014). Essai préliminaire d'élevage d'Anopheles labranchiae, vecteur du paludisme au Maroc

19. MATEE. (2002). arrêté conjoint du ministre de l'équipement du ministre charge de l'aménagement du territoire de l urbanisme de l habitat et de l environnement n 1275-02 17 octobre 2002. 
20. Messai ,N., Berchi,S., Boulknafd, F. , \& Louadi, K. (2011) . Inventaire, systématique et diversité biologique de Culicidae (Diptera

: Nematocera) dans la région de Mila (Algérie). Faun. Entomol., 63 (3): $203-206$.

21. Ministre de l'Equipement et du Ministre Charge de l'Amenagement du Territoire, de l'Urbanisme, de l'Habitat et de d'Environnement $\mathrm{N}^{\circ}$ 1275-02 du 17 Octobre 2002

22. RGPH Maroc . (2004) : Caractéristiques démographiques et socioéconomiques de la région du Gharb-Chrarda-Beni Hssen

23. Rodhain, F., Perez, C. (1985). Précis d'entomologie médicale et vétérinaire. Paris: Maloine $458 \mathrm{p}$.

24. Rodhain, F. ( 2000). Modifications climatiques : impacts potentiels sur la santé. L'exemple des maladies à vecteurs. Bull. Ass. Anc. Elèves Inst. Pasteur, 162: 8-14

25. Serghini, A., et al. (2010). Caractérisation hydrochimique d'un site Ramsar: Complexe

26. des zones humides de mohammedia: Maroc. Bulletin de l'institut scientifique, Rabat, section

27. Sciences de la vie, $\mathrm{n}^{\circ} 32(2), 133-145$

28. Savage, H.M., et Miller, B. (1995). House Mosquitoes of the U.S.A., Culex pipiens Complex. Wing Beats

29. Tran, A., et al. (2005). Modélisation des maladies vectorielles. Epidémiologie et santé animale, 47 : 35-51.

30. Trari,B., Dakki, M., Himmi,O. , \& Al Agbani, M.A . (2002). Les moustiques (Diptera-Cuicidae) du Maroc. Revue bibliographique (1916-2001) et inventaire des espèces. Bull. Soc. Pathol. Exot., 95 (4): 329 - 334.

31. World Health Organization. (2009). World Paludisme Report 
Planche $\mathrm{N}^{\circ} 1$ : Larve d'Anophele Labranchiae
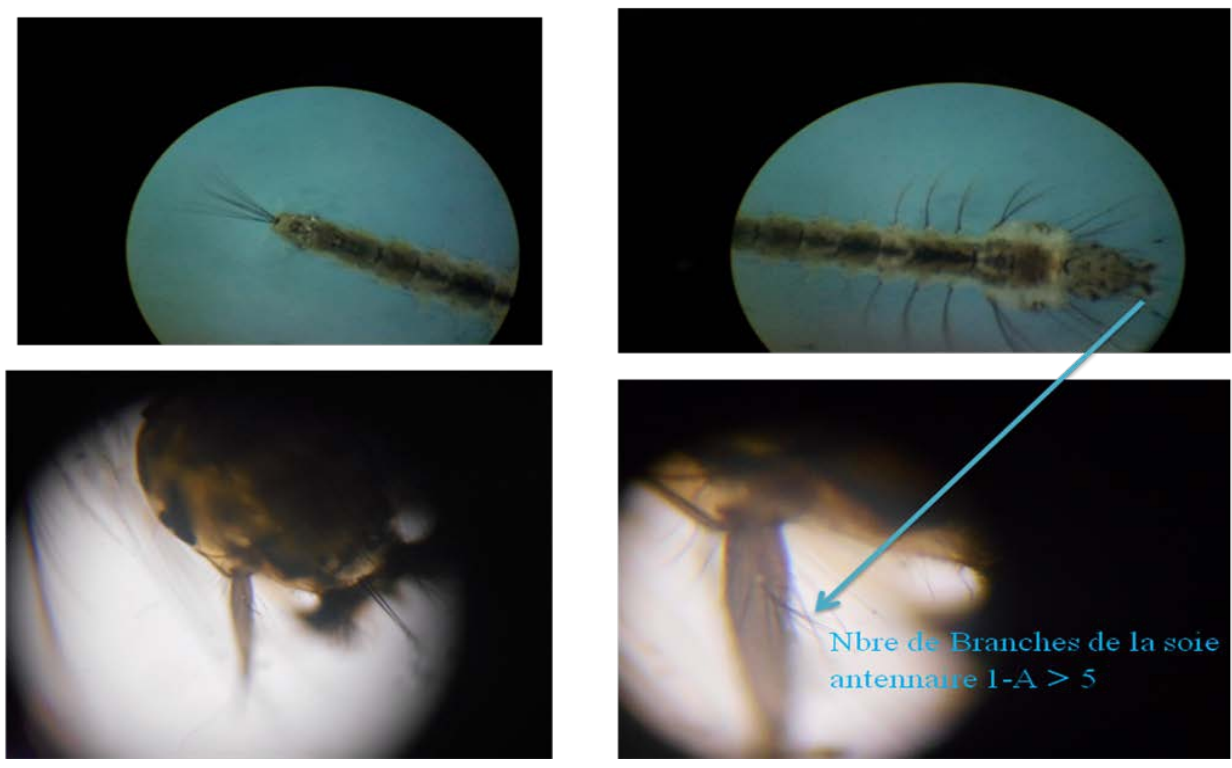

Planche $\mathrm{N}^{\circ} 2$ : Larve de Culex Pipiens
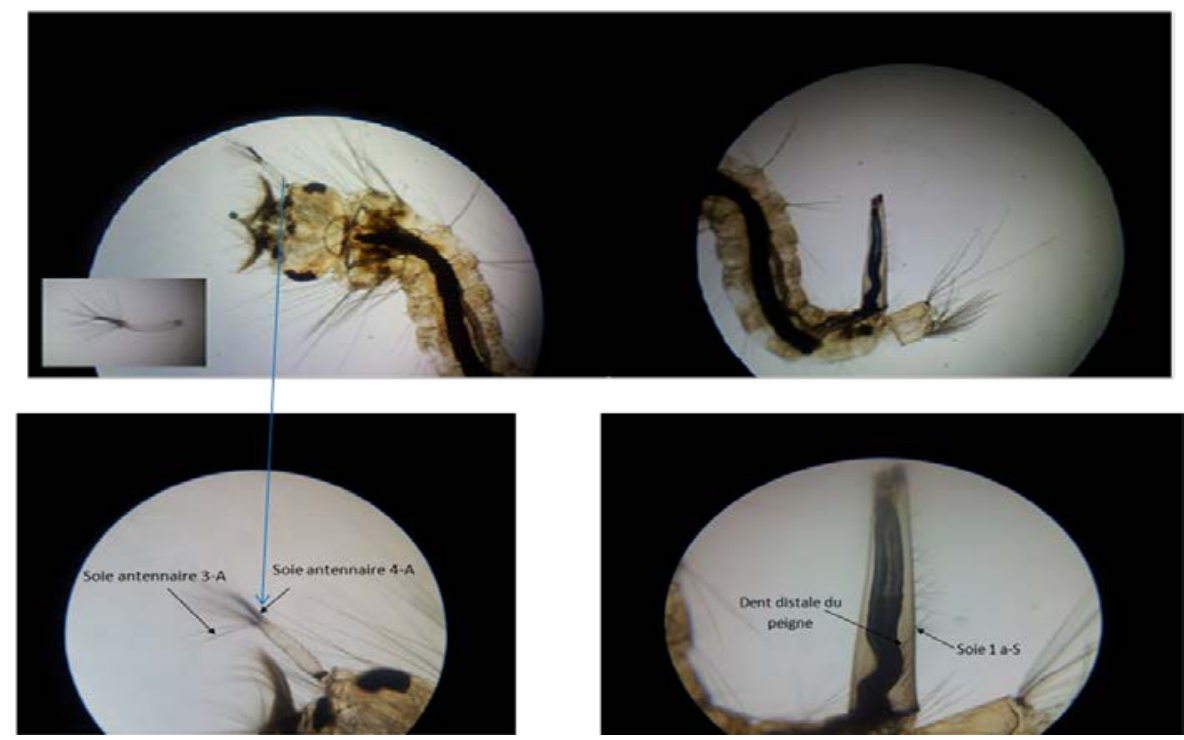